\title{
Synthesis and Characterization of Novel Hydantoins as Potential COX-2 Inhibitors: 1,5-Diarylhydantoins
}

\author{
Hae-Sun Park, Hee-Jeon Choi, Hea-Soon Shin, Sang Kook Lee, and Myung-Sook Park \\ College of Pharmacy, Duksthg Women's Lnwersity, Seoul 132-714, Korea. "E-mail msparkälukstmg ac.kr \\ College of Pharmacy Ewha Womans Lniversity, Seoul 120-750, Korea \\ Received Februarv 21, 2007
}

\begin{abstract}
To develop new COX-2 inhibitors. 1.5-diarylhydantoins and 1.5-diaryl-2-thiohydantoins were synthesized from phenylacetic acids by esterification bromination. $\mathrm{C}-\mathrm{N}$ bond formation and cyclization. Esters 1-3 were efficiently synthesized from the starting materials by reflux in absolute methanol for $3 \mathrm{~h}$ containing concentrated sulfuric acid as catalyst. Bromination was carried out with $N$-bromosuccinimide at $\mathrm{rt}$ in dichloromethane. Bromides 4-6 were reacted with aniline. $p$-anisidine. sulfanilamide in ethanol (or NA-dimethylformamide) to provide the amines 7-15. Hydantoins and 2-thiohydantoins 16-46 were synthesized from amines 7-15 by treating them with potassium isocranate (or potassium thiocyanate) and triethylamine. The synthetic process from alkyl $\alpha$-anilinophenylacetate 7-15 to 3-alkyllhydantoins was carried out in a one-pot reaction using alkyl isocyanate (alkyl isothiocyanate).
\end{abstract}

Key Words : Diary llhydantoins, Diaryl-2-thiohydantoins. Hỵdantoins, Synthesis, COX-2 inlubitors

\section{Introduction}

Nonsteroidal anti-inflammatory drugs (NSAIDs) are widely utilized for the treatment of inflammation. pain. and fever. but their use is often accompanied by gastrointestinal (GI) ulcerations and bleeding. ' Inflammatory processes are provoked by different chemicals/biospecies including proinflammatory enzymes/cytokines. small molecular species. such as eicosanoids. and tissue degrading enzymes. Of these factors cyclooxygenase (COX) catalyzes the conversion of arachidonic acid to prostaglandins (PGs) - key proinflammatory eicosanoids. All NSAIDs are believed to disrupt the biosynthesis of PGs by inhibiting the COX. ${ }^{2}$ In 1990s. Fu et al. discovered the existence of two isoforms of this enzyme. ${ }^{3}$ COX-1 a constitutive form and COX-2 an inducible form. COX-1 is expressed in normal tissues and is physiologically important for GI and renal functions. while the COX-2 isoform is located primarily in inflamed tissues ${ }^{4.7}$ It seems reasonable that a selective COX-2 inhibitor could block PGs production at a site of inflammation without affecting beneficial PGs in normal tissues. such as. in the stomach and kidney

The above hypothesis was partially proven when the first selective compounds. NS-398 and DuP-697, were tested in animal models. Both compounds showed anti-inflanumatory. analgesic and antipyretic activities. but they did not cause gastrointestinal lesions at ligh doses. ${ }^{8-10}$ Also. a new generation of anti-inflammmatory drugs. celecoxib (Celebrex $\left.{ }^{2}\right)^{.1}$ rofecoxib (Vioxx $\left.{ }^{(\bar{B}}\right)^{12}$ and valdecoxib $\left(\text { Bextra }^{\mathrm{B}}\right)^{1.3}$ are being widely prescribed to treat acute or chronic inflammation by providing symptomatic pain relief. COX-2 versus COX-1 selectivity demonstrated the superiority of these compounds over other NSAIDs in terms of reducing GI side effects. ${ }^{1+}$

However, emerging evidence suggests that adverse reactions such as GI irritation or ulceration and renal complications are associated with the prolonged use of $\mathrm{COX}-2$ selective inhibitors. These adverse reactions have been attributed. at least in part. to COX-1 inhibition during longterm exposure or at higher doses. ${ }^{15}$ COX-2 selective inhibitors are also known to suppress the synthesis of prostacyclin. a potent vasodilator. gastroprotectant. and platelet inhibitor. by inhibiting endothelial COX-2. Moreover. COX2 selective inhibitors do not inhibit the production of thromboxane. a vasoconstrictor. and promoter of platelet aggregation. which is sỵthesized in platelets by COX-1. ${ }^{13}$ Therefore. COX-2 selective inhibitors intrinsically lack antithrombotic activity. and some cardiovascular complications were associated with their use preclinically. ${ }^{17}$ Thus there is still a need for novel. selective potent COX-2 inhibitors with a better pharmacologic profile than the current $\mathrm{COX}-2$

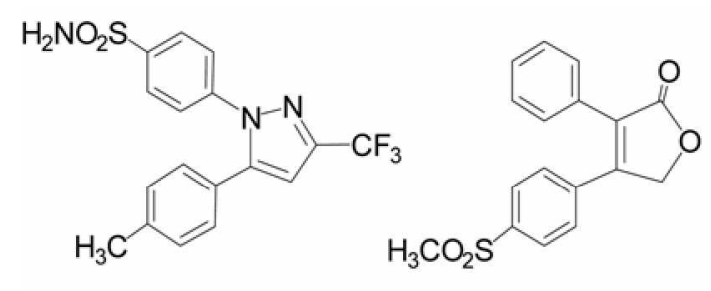

Celecoxib<smiles>Cc1onc(-c2ccccc2)c1-c1ccc(S(N)(=O)=O)cc1</smiles>

Valdecoxib<smiles>[R]C1=C([R])N(c2ccc([R])cc2)C(c2ccc([X])cc2)C1=O</smiles>

1,5-Diarylhydantoins 
inlibitors.

Due to the NSAIDs exhibited by selective COX-2 inhibitors, many researchs were reported during last several years. Very recently. Hwang et al., ${ }^{18}$ reported the indole derivatives as potential $\mathrm{COX}-2$ inhibitors

Here. we describe the synthesis of a novel series of hydantoin containing a 5 -niembered heterocyclic ring. In this study. we introduced a $p$-substituted phenyl group onto the 1- and 5-positions of the hydantoin ring in the hope of producing a selective COX-2 inhibitory effect. This study describes the syntheses of novel 1.5-diarylhydantoin derivatives with a phenyl group at the 5-position and a phenyl group (or $p$-sulfamylphenyl. $p$-methoxyphenyl group) at the 1-position (Scheme 1).

\section{Results and Discussion}

In Table 1. we summarized the physical properties and the optinal condition for the compounds 16-46. Two synthetic routes were classified as follows.

Production of hydantoin ring using $\mathrm{KNCO} / \mathrm{KNCS}$ (Class I). We have previously reported some preliminary

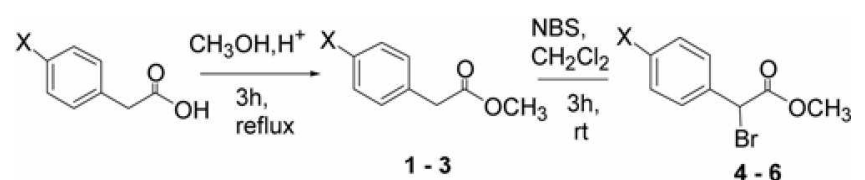

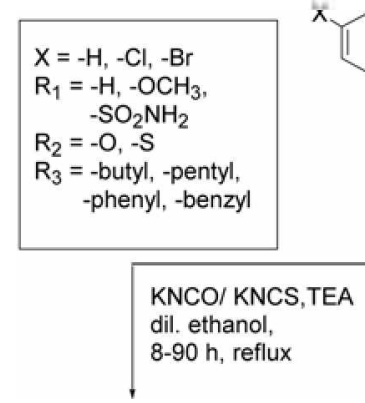

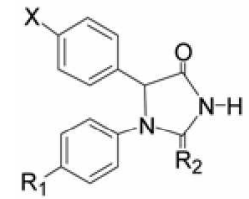
$33,38,40,42 \cdot 46$
$16-18,20-23,28$,

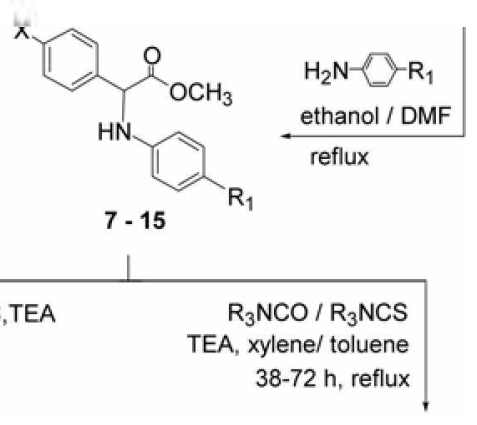<smiles>[R]c1ccc(C2C(=O)N([R3])C([R2])N2c2ccc([X])cc2)cc1</smiles>

$19,24-27,29-32$ $34-37,39,41$
Scheme 1. Synthesis of diaryl hydantoin derivatives 16-46.

Table 1. Target Connpounds 16-46 and Optimal Conditions for Synthesis

\begin{tabular}{|c|c|c|c|c|c|c|c|c|c|}
\hline Comp & $\begin{array}{l}\text { Class } / \\
\mathrm{I}^{a}, \mathrm{II}^{b}\end{array}$ & $\mathrm{R}_{\mathrm{l}}$ & $\mathrm{R}_{\text {. }}$ & $\mathrm{R}_{3}$ & $X$ & $\begin{array}{l}\text { Molar Ratió } \\
\text { Reag./Subs. }\end{array}$ & Time $/ \mathrm{hr}$ & $m p /{ }^{\circ} \mathrm{C}$ & Yield $/ \%{ }^{d}$ \\
\hline 16 & I & $\mathrm{H}$ & 0 & $\mathrm{H}$ & $\mathrm{H}$ & 1 & 8 & 190 & 59.1 \\
\hline 17 & I & $\mathrm{H}$ & 0 & $\mathrm{H}$ & $\mathrm{Cl}$ & 1.5 & 67 & $203.2-204.9$ & 81.0 \\
\hline 18 & I & $\mathrm{H}$ & 0 & $\mathrm{H}$ & $\mathrm{Br}$ & 1.5 & 48 & 209 & 79.8 \\
\hline 19 & II & $\mathrm{H}$ & 0 & n-buttyl & $\mathrm{H}$ & 1 & 38 & oil & - \\
\hline 20 & I & $\mathrm{H}$ & $\mathrm{S}$ & $\mathrm{H}$ & $\mathrm{H}$ & 1.8 & 32 & 189 & 59.7 \\
\hline 21 & I & $\mathrm{H}$ & S & $\mathrm{H}$ & $\mathrm{Cl}$ & 1 & 74 & $205.1-205.9$ & 68.2 \\
\hline 22 & I & $\mathrm{H}$ & $\mathrm{S}$ & $\mathrm{H}$ & $\mathrm{Br}$ & 1 & 90 & 205 & 75.2 \\
\hline 23 & I & $\mathrm{CH}_{3} \mathrm{O}$ & 0 & $\mathrm{H}$ & $\mathrm{H}$ & 1.5 & 8 & $193.7-195.5$ & 77.9 \\
\hline 24 & II & $\mathrm{CH}_{3} \mathrm{O}$ & 0 & n-butỵl & $\mathrm{H}$ & 2 & 48 & $101.8-103.7$ & 62.3 \\
\hline 25 & II & $\mathrm{CH}_{3} \mathrm{O}$ & 0 & n-pentyl & $\mathrm{H}$ & 2 & 48 & $122.4-125.3$ & 41.3 \\
\hline 26 & II & $\mathrm{CH}_{3} \mathrm{O}$ & 0 & plentel & $\mathrm{H}$ & 2 & 48 & $145.8-1480$ & 32.3 \\
\hline 27 & II & $\mathrm{CH}_{3} \mathrm{O}$ & 0 & benzyl & $\mathrm{H}$ & 2 & 48 & $118.9-121.6$ & 55.2 \\
\hline 28 & I & $\mathrm{CH}_{3} \mathrm{O}$ & 0 & $\mathrm{H}$ & $\mathrm{Cl}$ & 2 & 24 & $197.6 \cdot 200.4$ & 90.4 \\
\hline 29 & II & $\mathrm{CH}_{3} \mathrm{O}$ & 0 & n-butyl & $\mathrm{Cl}$ & 2 & 72 & $93.6-95.2$ & 67.2 \\
\hline 30 & II & $\mathrm{CH}_{2} \mathrm{O}$ & 0 & n-pentyl & $\mathrm{Cl}$ & 2 & 48 & $97.4-98.6$ & 79.4 \\
\hline 31 & II & $\mathrm{CH}_{3} \mathrm{O}$ & 0 & phenyl & $\mathrm{Cl}$ & 2 & 48 & $180.2-183.2$ & 62.1 \\
\hline 32 & II & $\mathrm{CH}_{3} \mathrm{O}$ & 0 & benzy] & $\mathrm{Cl}$ & 2 & 48 & $147.6-148.8$ & 89.6 \\
\hline 33 & I & $\mathrm{CH}_{3} \mathrm{O}$ & 0 & $\mathrm{H}$ & $\mathrm{Bt}$ & 2 & 24 & $194.5-195.9$ & 93.1 \\
\hline 34 & II & $\mathrm{CH}_{3} \mathrm{O}$ & 0 & n-butyl & $\mathrm{Bt}$ & 2 & 72 & $102.7-104.5$ & 56.7 \\
\hline 35 & II & $\mathrm{CH}_{3} \mathrm{O}$ & 0 & n-pentyl & Er & 2 & 48 & $91.7-93.9$ & 54.8 \\
\hline 36 & II & $\mathrm{CH}_{3} \mathrm{O}$ & 0 & plienyl & $\mathrm{Br}$ & 2 & 48 & $189.5-190.1$ & 30.4 \\
\hline 37 & II & $\mathrm{CH}_{3} \mathrm{O}$ & 0 & benzyl & $\mathrm{Br}$ & 2 & 48 & $139.5-1+10$ & 87.7 \\
\hline 38 & I & $\mathrm{CH}_{3} \mathrm{O}$ & $\mathrm{S}$ & $\mathrm{H}$ & $\mathrm{H}$ & 2 & 16 & $197.7-200.5$ & 92.7 \\
\hline 39 & II & $\mathrm{CH}_{3} \mathrm{O}$ & S & benzy! & $\mathrm{H}$ & 2 & 48 & $119.1-121.1$ & 48.6 \\
\hline 40 & I & $\mathrm{CH}_{2} \mathrm{O}$ & $S$ & $\mathrm{H}$ & $\mathrm{Br}$ & 2 & 60 & $184.6-186.8$ & 93.3 \\
\hline 41 & II & $\mathrm{CH}_{2} \mathrm{O}$ & $\mathrm{S}$ & benzy] & $\mathrm{Br}$ & 2 & 48 & $188.7-191.0$ & 27.2 \\
\hline 42 & I & $\mathrm{SO}_{2} \mathrm{NH}_{2}$ & 0 & $\mathrm{H}$ & $\mathrm{H}$ & 2 & 16 & $202.0-211.5$ & 60.0 \\
\hline 43 & I & $\mathrm{SO}_{2} \mathrm{NH}_{2}$ & 0 & $\mathrm{H}$ & $\mathrm{Cl}$ & 2 & 16 & $212.4-225.0$ & 68.1 \\
\hline 44 & I & $\mathrm{SO}_{-} \mathrm{NH}_{2}$ & 0 & $\mathrm{H}$ & $\mathrm{Br}$ & 2 & 18 & $221.2-226.0$ & 95.8 \\
\hline 45 & I & $\mathrm{SO} . \mathrm{NH}_{2}$ & $\mathrm{~S}$ & $\mathrm{H}$ & $\mathrm{H}$ & 2 & 30 & $242.0-248.0$ & 76.9 \\
\hline 46 & I & $\mathrm{SO}_{-} \mathrm{NH}_{2}$ & $\mathrm{~S}$ & $\mathrm{H}$ & $\mathrm{Cl}$ & 2 & 50 & $2009-2042$ & 69.3 \\
\hline
\end{tabular}

"At All reactions were performed in ethanol xvlene under reflux, except 19 (in toluene). Reag subs is the ratio of reagent (KNCO. KNCS. R3NCO. and $\mathrm{R}_{2} \mathrm{NCS}$ ) to substrate (amine 7-15). ${ }^{\text {tY }}$ Yields reterred to isolated product. 
results for a novel series of $\alpha$-amino acids $7-15^{19}$ (Scheme 1). The classical method for the preparation of hydantoins involves the reaction of $a$-anino acids with potassium cyanate: this reaction was carried out in boiling aqueous solution. The free hydantoic acid produced may be isolated by acidification of the solution or it may be converted into the corresponding hydantoin by treatment with $25 \% \mathrm{HCl}$.

This cyclization was preferentially carried out by refluxing a solution in $d i$-ethanol. It should be noted that the production of analogues of 2-thiohydantoin needs additional mild base such as triethylamine, because of the different reactivity of sulfur versus oxygen containing analogs. The formation of hydantoin was generally faster than that of 2 . thiohydantoins. In some cases (e.g., $\mathrm{R}_{1}=$ anilino group or $p$ methoxyanilino group), the reaction mixture was stirred for $\mathrm{l} \mathrm{h}$ in order to increase the yield of Class I after adding $\mathrm{c}$ $\mathrm{HCl}$. In other case (e.g., $\mathrm{R}_{1}=p$-sulfanylanilino group). the $\mathrm{pH}$ of the reaction nixture was optimized with respect to yield according to the presence of halogen $(\mathrm{X}=\mathrm{H}, \mathrm{Cl} . \mathrm{Br})$. For example. compounds $\mathbf{4 2}$ and $\mathbf{4 5}$ were obtained in maximal yields at $\mathrm{pH} 4$. while compounds 43,44 , and +6 required a $\mathrm{pH} \mathrm{I}$. Also. conpounds containing a halogen $(\mathrm{X}=$ $\mathrm{Cl} . \mathrm{Br}$ ) required longer reaction times than those without a halogen (Table 1).

Identification of Class I products was performed by NMR. IR and GC-MS. In the ${ }^{1} \mathrm{H}-\mathrm{NMR}$, the NH peak disappeared whereas the $\mathrm{CH}$ peak appeared as a singlet at $5 \mathrm{ppm}$. In the IR spectra. the carbonyl absorption band was not present at $1700 \mathrm{~cm}^{-1}$ because of the tautomeric nature of the hydantoin ring (Figure 1). When $\mathrm{R}_{1}$ was $\mathrm{H}$ or $\mathrm{CH}_{3} \mathrm{O}$. this band appeared clearly. We believed that the enol-form was more stable than keto-form because both of functional groups donated an electron to the hydantoin ring.

Production of hydantoin ring using RNCO/RNCS (onepot reaction) (Class II). A large number of aryl and alkyl isocyanate and isothiocyanates have been employed in this reaction. ${ }^{3(1)}$ The $N$-aralkylhydantoin analogues containing a phenyl or methoxyphenyl at position 1 of the hydantoin ring ( $\mathrm{R} 3=n$-buty l, $n$-pentyl phenyl, benzyl) were synthesized in a one-step process (Scheme 1) from $\alpha$-anino acids 7-15 using aralkyl isocyanate or isothiocyanate in xylene using a mild base like triethylamine. ${ }^{31}$ Molecular sieve $(+\AA)$ was added to renove the methanol produced. Reaction rates were found to be highly dependent on the solvent sy'stem used.

The reactivity of aralkyl isothiocyanate was low versus isocyanate and it generated many side reactions. The success or failure of the reaction depended on the nature of the functional group ( $\mathrm{RI}=p$-methoxy or $p$-sulfonamide). For example. when $\mathrm{Rl}$ was $p$-methoxy (an electron donating group). the reaction proceeded well to give the desired products in good yield (Table 1). On the other hand. when $\mathrm{RI}$ was $p$-sulfonamide group (an electron withdrawing group). the reaction did not proceed well. Also. compounds with $p$-sulfonamide did not react regardless of solvent type (e.g. xylene, DMF, toluene, ethanol. etc.).

In the ${ }^{1} \mathrm{H}-\mathrm{NMR}$ spectra, the $-\mathrm{CH}$ peak was shifted from 5 $\mathrm{ppm}$ to $6 \mathrm{ppm}$. Also in the IR spectra, two carbonyl absorption bands appeared at $1700 \mathrm{~cm}^{-1}$. whereas they were absent in Class I (Table 1). We considered that the keto-form was more stable than the enol-form in the case of substitution aralkyl group at the 3-position of the hydantoin ring

In conclusion, we have developed a new class of 1.5diarylhydantoins. which is the hybrid type compound 16-46 by structural modification of celecoxib and rofecoxib. 1.5Diarylhydantoins were synthesized by esterification, bromination, $\mathrm{C}-\mathrm{N}$-bond formation and cyclization.

\section{Experimental Section}

Chemicals. Chemicals were supplied by Aldrich. Sigma, Merck. and Tokyo Kasei. Melting points were determined in open capillary tubes on a Büchi 535 melting point apparatus and uncorrected. NMR spectra were recorded using a Bruker $300 \mathrm{MHz}$ NMR spectrometer. Chemical shifts are reported in parts per million and were recorded in chloroform-d or dimethyl-d $\mathrm{d}_{i}$ sulfoxide with tetramethylsilane as the internal standard. NMR spin multiplicities are indicated by the symbols: $\mathrm{s}$ (singlet), $\mathrm{d}$ (doublet), $\mathrm{t}$ (triplet), $\mathrm{q}$ (quartet), and $\mathrm{m}$ (multiplet). IR spectra were recorded on a Perkin-Elmer l6F PC. FT-IR spectrometer using $\mathrm{NaCl}$ discs and pellets. Mass fragmentations were recorded using an Agilent $6890 \mathrm{GC}$ and $5973 \mathrm{MS}$

General procedure for the compounds 16-18, 23, 28, 33 and 42-44. Methyl $\alpha$-anilinophenylacetate 7 (2.4 g. 10 mumol) was dissolved in ethanol $(20 \mathrm{~mL})$ by heating. KNCO $(0.81 \mathrm{~g} .10 \mathrm{mmol})$ in water $(10 \mathrm{~mL})$ was added quickly and then refluxed for $8 \mathrm{~h}$. The reaction mixture was concentrated under reduced pressure to $3 \mathrm{~mL}$ and $\mathrm{c}-\mathrm{HCl}$ was added to $\mathrm{pH}$ 1 in an ice-bath. The resulting solid was washed with icewater and recrystallized in $d i$-ethanol.

1,5-Diphenylhydantoin (16): Yield: $1.49 \mathrm{~g}(59.1 \%) . \mathrm{mp}$ $190^{\circ} \mathrm{C}$. TLC [methylene chloride methanol (9:1)] Rf 0.27 . ${ }^{1} \mathrm{H}$ NMR (DMSO- $d_{6}$ ) $\delta 7.5 \mathrm{l}$ (d. $J=6.0 \mathrm{~Hz} .2 \mathrm{H}$. aromatic), $7.39-7.26$ (m. $3 \mathrm{H}$, aromatic), 7.03 (t. $J=9.0 \mathrm{~Hz} .2 \mathrm{H}$, aromatic). $6.65(\mathrm{~d} . J=6.0 \mathrm{~Hz} .2 \mathrm{H}$. aromatic). 6.54 (t. $J=9.0$
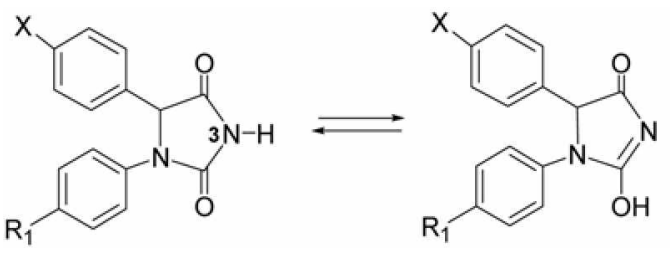<smiles>[X]c1ccc(-c2c(O)[nH]c(=O)n2-c2ccc(Br)cc2)cc1</smiles><smiles>[R]c1ccc(-c2c(O)nc(O)n2-c2ccc([X])cc2)cc1</smiles>

Figure 1. Keto-enol tautomerizations of hydantoin ring. 
$\mathrm{Hz}, 1 \mathrm{H}$, aromatic) $5.07(\mathrm{~s}, 1 \mathrm{H}, \mathrm{CH}), 3.34$ (s. $1 \mathrm{H} . \mathrm{NH}),{ }^{13} \mathrm{C}$ NMR (DMSO- $\left.d_{6}\right) \delta 172.86(\mathrm{C}=\mathrm{O} \times 2)$. 146.86 .138 .45 . 128.63. 128.34, 127.65. 127.40, 116.43, 112.93 (aromatic $\times$ 2), $59.57(\mathrm{CH}), 55.09\left(\mathrm{CH}_{3}\right)$. FT-IR $(\mathrm{KBr}) \mathrm{cm}^{-1} 3408(\mathrm{NH})$. $2991(\mathrm{CH})$. GC-MS: ni/z $252.32\left(\mathrm{M}^{+}\right)$. 180.1 (100.0), 181.1 $(87.18), 77.1(35.0)$. $182.1(11.8), 51.1(10.6)$.

1-Phenyl-5-(p-chlorophenyl)hydantoin (17): Yield: $81.0 \%$. mp 203.2-204.9 ${ }^{\circ} \mathrm{C}$, TLC [methylene chloride: methanol (9:1)] Rf 0.28. ${ }^{\mathrm{H}} \mathrm{H}$ NR (DMSO- $d_{6}$ ) $\delta 7.52$ (d. $J=$ $8.7 \mathrm{~Hz} .2 \mathrm{H}$. aronatic), 7.42 (d. $J=8.4 \mathrm{~Hz} .2 \mathrm{H}$. aromatic). $7.03(\mathrm{t}, J=8.4 \mathrm{~Hz}, 2 \mathrm{H}$. aromatic). $6.64(\mathrm{~d}, J=7.8 \mathrm{~Hz}, 2 \mathrm{H}$. aromatic), 6.57 (t. $J=7.2 \mathrm{~Hz} .1 \mathrm{H}$, aromatic), 5.13 (s, $\mathrm{lH}$. CH). $3.35(\mathrm{~s}, 1 \mathrm{H}, \mathrm{NH}) .{ }^{13} \mathrm{C}$ NMR (DMSO- $\left.d_{6}\right) \delta 172.89$ $(\mathrm{C}=\mathrm{O} \times 2), 147.07,138.16,132.63 .129 .69 .129 .10 .128 .75$. $117.02,113.47$ (aromatic $\times 2) .59 .31(\mathrm{CH})$. FT-IR $(\mathrm{KBr}) \mathrm{cm}^{-1}$ $3412(\mathrm{NH}), 2993(\mathrm{CH}) .770(\mathrm{C}-\mathrm{Cl})$. GC-MS: $\mathrm{m} / \mathrm{z} 287.76$ $\left(\mathrm{M}^{+}\right) .214 .1(100.0) .215 .1(94.5) .77 .1(47.1), 216.0(43.6)$. $217.0(32.0)$.

1-Phenyl-5-(p-bromophenyl)hydantoin (18): Yield: $79.8 \%$. mip $209{ }^{\circ} \mathrm{C}$, TLC [methylene chloride:methanol (9:1)] Rf 0.34. 'H NMR (DMSO- $\left.d_{6}\right) \delta 7.56(\mathrm{~d}, J=8.4 \mathrm{~Hz}$. $2 \mathrm{H}$. aromatic). 7.47 (d. $J=8.7 \mathrm{~Hz}, 2 \mathrm{H}$. aromatic). 7.03 (t. $J=$ $8.4 \mathrm{~Hz} .2 \mathrm{H}$. aromatic), 6.64 (d. $J=7.8 \mathrm{~Hz} .2 \mathrm{H}$. aromatic). $6.55(\mathrm{t}, J=7.2 \mathrm{~Hz}$. IH. aromatic), 5.12 (s. IH. CH), 3.36 (s. $1 \mathrm{H}, \mathrm{NH}) .{ }^{13} \mathrm{C}$ NMR (DMSO- $\left.d_{6}\right) \delta 172.83(\mathrm{C}=\mathrm{O} \times 2), 147.05$. 138.56. 131.68, 130.05. 129.10, 121.22. 117.04, 113.48 (aronatic $\times 2), 59.35(\mathrm{CH})$. FT-IR $(\mathrm{KBr}) \mathrm{cm}^{-1} 3406(\mathrm{NH})$. $2992(\mathrm{CH}) .766(\mathrm{C}-\mathrm{Br})$. GC-MS: m/z $332.16\left(\mathrm{M}^{-}\right), 169.0$ $(100.0), 171.0(99.2), 261.0(73.4) .263 .0(69.7) .90 .1(30.8)$.

1-(p-Methoxy]henyl)-5-phenylhydantoin (23): Yield: $77.9 \%$. nip 193.7-195.5 ${ }^{\circ} \mathrm{C}$. TLC [n-hexane:ethyl acetate (2:1)] Rf 0.1. [methylene chloride methanol (9:1)] Rf 0.29 . ${ }^{1} \mathrm{H}$ NMR (DMSO- $d_{6}$ ) $\delta 7.50$ (d, $J=7.2 \mathrm{~Hz}, 2 \mathrm{H}$. aromatic). 7.37-7.25 $(\mathrm{m}+\mathrm{d}, 3 \mathrm{H}$, aromatic). $6.64(\mathrm{~d}+\mathrm{d} . J=9.3$ and $9 \mathrm{~Hz}$. $4 \mathrm{H}$. aromatic), 5.01 (s. IH. CH). $3.60\left(\mathrm{~s}, 3 \mathrm{H}, \mathrm{CH}_{3}\right), 3.40$ (s. 1H, NH) ${ }^{13} \mathrm{C}$ NMR (DMSO- $\left.d_{6}\right) \delta 173.00(\mathrm{C}=\mathrm{O} \times 2), 151.04$. 140.96. 138.75, 128.26. 127.51, 127.35. 114.27, 114.10 (aromatic $\times 2), 60.42(\mathrm{CH}), 55.09\left(\mathrm{CH}_{\hat{j}}\right)$. FT-IR $(\mathrm{KBr}) \mathrm{cm}^{-1}$ $3402(\mathrm{NH}), 151+(\mathrm{CO})$. GC-MS: $\mathrm{m} / \mathrm{z} 282.30\left(\mathrm{M}^{-}\right), 196.1$ (100.00). $211.1(88.27) .167 .1$ (21.76). $210.2(15.50) .197 .1$ (15.11).

1-(p-Methoxyphenyl)-5-(p-chlorophenyl)hydantoin (28): Yield: $90.4 \%$ mp $197.6-200.4{ }^{\circ} \mathrm{C}$. TLC [n-hexane:ethyl acetate (2:1)] Rf 0.1, [methy lene chloride:methanol (9:1)] Rf 0.22 . ${ }^{1} \mathrm{H}$ NMR (DMSO- $d_{6}$ ) $\delta 7.52$ (d. $J=8.4 \mathrm{~Hz}, 2 \mathrm{H}$. aromatic), $7.4 \mathrm{l}(\mathrm{d}, J=8.4 \mathrm{~Hz}, 2 \mathrm{H}$, aromatic), $6.63(\mathrm{~d}+\mathrm{d} . J=$ 9 and $9 \mathrm{~Hz}, 4 \mathrm{H}$ aromatic) 5.06 (s. $1 \mathrm{H}, \mathrm{CH}$ ). 3.60 (s. $3 \mathrm{H}$. $\mathrm{CH}_{j}$ ). 3.34 (s. $1 \mathrm{H} . \mathrm{NH}$ ). ${ }^{13} \mathrm{C}$ NMR (DMSO-do) $\delta 172.62$ $(\mathrm{C}=\mathrm{O} \times 2), 151.14,140.66,137.87 .132 .10 .129 .22 .128 .24$. $114.25,114.22$ (aromatic $\times 2), 59.59(\mathrm{CH}), 55.14\left(\mathrm{CH}_{3}\right) . \mathrm{FT}-$ IR $(\mathrm{KBr}) \mathrm{cm}^{-1} 340 \mathrm{I}(\mathrm{NH}), 15 \mathrm{l} 4(\mathrm{CO}) .769$ (C-Cl). GC-MS: $\mathrm{m} / \mathrm{z} 316.74\left(\mathrm{M}^{-}\right), 230.0(100.00) .245 .0 \quad(91.43) .232 .0$ (34.32), 247.0 (30.96). 246.0 (18.70).

1-(p-Methoxyphenyl)-5-(p-bromophenyl)hydantoin (33): Yield: $93.1 \%$ mp 194.5-195.9 ${ }^{\circ} \mathrm{C}$. TLC [n-hexane:ethy] acetate $(2: 1)$ ] Rf 0.1 , [methy lene chloride:nethanol $(9: 1)$ ] Rf 0.17 . ${ }^{1} \mathrm{H}$ NMR (DMSO-d ) $\delta 7.55$ (d. $J=8.4 \mathrm{~Hz}, 2 \mathrm{H}$. aromatic). 7.45 (d, $J=8.4 \mathrm{~Hz}, 2 \mathrm{H}$. aromatic), $6.63(\mathrm{~d}+\mathrm{d}, J=$ 9 and $9 \mathrm{~Hz}, 4 \mathrm{H}$. aromatic). $5.06(\mathrm{~s}, \mathrm{LH} . \mathrm{CH}), 3.60$ (s. $3 \mathrm{H}$, $\mathrm{CH}_{3}$ ). 3.36 (s. $1 \mathrm{H}, \mathrm{NH}$ ). ${ }^{13} \mathrm{C}$ NMR (DMSO- $d_{\text {) }} \delta 172.55$ $(\mathrm{C}=\mathrm{O} \times 2) .151 .14,140.63 .138 .28 .131 .16 .129 .58,120.67$. 114.32. 114.24 (aromatic $\times 2), 59.62(\mathrm{CH}) .55 .06\left(\mathrm{CH}_{3}\right)$. FTIR $(\mathrm{KBr}) \mathrm{cm}^{-1} 340 \mathrm{l}(\mathrm{NH}), 1513$ (CO). 765 (C-Br). GC-MS: $\mathrm{ml} / \mathrm{z} 361.20\left(\mathrm{M}^{-}\right), 291.0$ (100.00). 289.0 (99.18). 274.0 (88.27). 275.9 (87.91), $290.0(27.41)$.

5-Phenyl-1-( $p$-sulfamylphenyl)hydantoin (42): Yield: $60.0 \%$. mp 202.0-211.5 ${ }^{\circ} \mathrm{C}$. TLC [methylene chloride: methanol (9:1)] Rf 0. 1. 'H NMR (DMSO- $\left.d_{6}\right) \delta 7.48$ (d. $J=$ $8.7 \mathrm{~Hz}, 4 \mathrm{H}$. aromatic), $7.31-7.18(\mathrm{~m}, 3 \mathrm{H}$. aromatic), $6.79(\mathrm{~s}$, $2 \mathrm{H}$. $\left.\mathrm{NH}_{2}\right) .6 .58$ (d. $J=8.7 \mathrm{~Hz}, 2 \mathrm{H}$, aromatic). 4.89 (s, $1 \mathrm{H}$, $\mathrm{CH}), 3.24$ (s. $1 \mathrm{H}, \mathrm{NH}$ ). ${ }^{13} \mathrm{C}$ NMR (DMSO- $\left.d_{6}\right) \delta 171.32$ $(\mathrm{C}=\mathrm{O} \times 2) .149 .63,140.05 .130 .05 .127 .89 .127 .03,126.94$. 126.71. 111.63 (aromatic $\times 2), 60.53(\mathrm{CH})$. FT-IR $(\mathrm{NaCl})$ $\mathrm{cm}^{-1} 3369(\mathrm{NH}), 3193\left(\mathrm{NH}_{2}\right), 1710(\mathrm{C}=\mathrm{O}) .1301\left(\mathrm{SO}_{2}\right) . \mathrm{GC}$. MS: $\mathrm{m} / \mathrm{z} 331.35\left(\mathrm{M}^{-}\right) .180 .1$ (100.00). $181.1(84.25), 77.1$ (36.22). 51.1 (11.75). 182.1 (11.11).

5-(p-Chlolophenyl)-1-(p-sulfamylphenyl)hydantoin (43): Yield: $68.1 \%, \operatorname{mp} 212.4-225.0^{\circ} \mathrm{C}$, TLC [methylene chloride: methanol (9:1)] Rf 0.1. 'H NMR (DMSO- $\left.c_{6}\right) \delta 7.80-7.43$ (m, $6 \mathrm{H}$, aromatic), $6.93\left(\mathrm{~s}, 2 \mathrm{H}, \mathrm{NH}_{2}\right), 6.75(\mathrm{~d} . J=8.7 \mathrm{~Hz}$, 2H. aromatic), 5.29 (d, $J=7.2 \mathrm{~Hz} . \mathrm{lH}, \mathrm{CH}$ ). 3.45 (s. $\mathrm{lH}$, $\mathrm{NH}) .{ }^{13} \mathrm{C}$ NMR (DMSO- $\left.c_{i}\right) \delta 172.22(\mathrm{C}=\mathrm{O} \times 2), 152.61$, 150.92, 137.30. 132.93. 129.69, 129.19, 128.94. 126.81, 112.23 (aromatic $\times 2), 58.72(\mathrm{CH})$. FT-IR $(\mathrm{NaCl}) \mathrm{cm}^{-1} 3332$ $(\mathrm{NH}), 3160\left(\mathrm{NH}_{2}\right), 1712(\mathrm{C}=\mathrm{O}) .1334\left(\mathrm{SO}_{2}\right), 722(\mathrm{C} \cdot \mathrm{Cl})$. GC-MS: $\mathrm{m} / \mathrm{z} 365.80\left(\mathrm{M}^{+}\right)$. $214.1(100.00), 215.0(95.39)$, 77.0 (46.56), $216.0(44.66), 217.0$ (32.02).

5-(p-Bromophenyl)-1-(p-sulfamylphenyl)hydantoin (44): Yield: $95.8 \% \cdot \operatorname{mp} 221.2-226.0^{\circ} \mathrm{C}$, TLC [methylene chloride :methanol (9:1)] Rf 0.1. ${ }^{1} \mathrm{H}$ NMR (DMSO- $d_{0}$ ) $\delta 7.60-7.44$ (m, 6H. aromatic). $6.92\left(\mathrm{~s}, 2 \mathrm{H}, \mathrm{NH}_{2}\right) .6 .74(\mathrm{~d} . J=9 \mathrm{~Hz} .2 \mathrm{H}$, aromatic). 5.27 (d, $J=7.2 \mathrm{~Hz}, 1 \mathrm{H}, \mathrm{CH}) .3 .43$ (s. lH, NH) ${ }^{13} \mathrm{C}$ NMR (DMSO- $\left.d_{6}\right) \delta 171.66(\mathrm{C}=\mathrm{O} \times 2) .152 .15 .150 .45$. $137.31,131.39 .129 .56,128.72 .126 .36,121.03,111.77$ (aromatic $\times 2$ ). $58.35(\mathrm{CH})$. FT-IR $(\mathrm{NaCl}) \mathrm{cm}^{-1} 3337(\mathrm{NH})$, $3159\left(\mathrm{NH}_{2}\right), 1711(\mathrm{C}=\mathrm{O}) .1336\left(\mathrm{SO}_{2}\right), 721(\mathrm{C}-\mathrm{Br}) . \mathrm{GC}-\mathrm{MS}$ : $\mathrm{m} / \mathrm{z}+10.25\left(\mathrm{M}^{-}\right), 221.1 \quad(100.00) .281 .0(82.28)$. 147.1 (71.86). 207.0 (65.85). 73.1 (57.75).

General procedure for the compounds $20-22,38,40$, 45-46. Methỵl $\alpha$-anilinophenylacetate 7 (1.2 g. $4.98 \mathrm{mmol})$ was dissolved in ethanol ( $10 \mathrm{~mL})$ by heating. KNCS $(0.87 \mathrm{~g}$. $8.95 \mathrm{~nm}$ ol) in water $(5 \mathrm{~mL})$ and trietlyy lamine $(1.26 \mathrm{~mL}$. $9.04 \mathrm{mmol}$ ) were added and then refluxed for $8 \mathrm{~h}$. The reaction mixture was then concentrated under reduced pressure to $3 \mathrm{~mL}$ and $\mathrm{c}-\mathrm{HCl}$ was added to $\mathrm{pH} 1$ in an icebath. The resulting solid was washed with ice-water and recrystallized in dil-ethanol.

1,5-Diphenyl-2-thiohydantoin (20): Yield: $0.8 \mathrm{~g} \mathrm{(59.7 \% )}$ mp $189^{\circ} \mathrm{C}$. TLC [methylene cliloride:methanol (9:1)] Rf 0.39 . ${ }^{\mathrm{l}} \mathrm{H}$ NMR (DMSO- $\left.c_{6}\right) \delta 7.51$ (d, $J=6.9 \mathrm{~Hz} .2 \mathrm{H}$, aromatic). $7.33(\mathrm{~m}, 3 \mathrm{H}$. aromatic), $7.03(\mathrm{t} . J=7.9 \mathrm{~Hz} .2 \mathrm{H}$, aromatic). 6.65 (d. $J=7.8 \mathrm{~Hz}, 2 \mathrm{H}$, aromatic). $6.54(\mathrm{t} . J=7.2$ $\mathrm{Hz} .1 \mathrm{H}$. aromatic). 5.07 (s. $1 \mathrm{H}, \mathrm{CH}), 3.34$ (s, $1 \mathrm{H}, \mathrm{NH}) .{ }^{13} \mathrm{C}$ NMR (DMSO- $\left.d_{6}\right) \delta 172.80(\mathrm{C}=\mathrm{O} . \mathrm{C}=\mathrm{S}) .146 .86 .138 .55$. 
128.61. 128.30. 127.58, 127.37. 116.39. 112.91 (aromatic 2), $59.65(\mathrm{CH})$. FT-IR $(\mathrm{NaCl}) \mathrm{cm}^{-1} 3275(\mathrm{NH}), 2928(\mathrm{CH})$. $171+(\mathrm{C}=\mathrm{O})$. GC.MS: $\mathrm{m} / \mathrm{z} 268.32\left(\mathrm{M}^{+}\right) .207 .0(100.0)$. 147.1 (73.1). $281.1(68.8) .73 .1(607)$.

1-Phenyl-5-(p-chlorophenyl)-2-thiohydantoin (21): Yield: $68.2 \%$. mp $205.1-205.9^{\circ} \mathrm{C}$. TLC [methylene chloride :methanol (9:1)] Rf 0.31. ${ }^{1} \mathrm{H}$ NMR (DMSO- $\left.d d_{6}\right) \delta 7.53$ (d. $J=$ $6.6 \mathrm{~Hz} .2 \mathrm{H}$. aronatic). 7.42 (d. $J=6.6 \mathrm{~Hz} .2 \mathrm{H}$. aromatic). $7.03(\mathrm{t}, J=7.5 \mathrm{~Hz}, 2 \mathrm{H}$. aromatic). $6.64(\mathrm{~d}, J=7.5 \mathrm{~Hz} .2 \mathrm{H}$. aromatic), 6.55 (t. $J=7.2 \mathrm{~Hz} .1 \mathrm{H}$, aromatic), $5.14(\mathrm{~s}, 1 \mathrm{H}$. $\mathrm{CH}) .3 .38(\mathrm{~s}, 1 \mathrm{H}, \mathrm{NH}),{ }^{13} \mathrm{C}$ NMR (DMSO- $\left.d_{6}\right) \delta 172.91$ $(\mathrm{C}=\mathrm{O} . \mathrm{C}=\mathrm{S}) .147 .07 .138 .11,132.66 .131 .55,129.70,129.11$. $128.77,117.04,113.48$ (aromatic $\times 2), 59.29(\mathrm{CH}) . \quad$ FT-IR $(\mathrm{KBr}) \mathrm{cm}^{-1} 3407(\mathrm{NH}), 2993(\mathrm{CH}) .770(\mathrm{C}-\mathrm{Cl}) . \mathrm{GC}-\mathrm{MS}$ : $\mathrm{m} / \mathrm{z} 303.82\left(\mathrm{M}^{-}\right), 125.0(100.0)$. 217.1 (63.8). $127.0(33.2)$. 219.1 (21.2). $216.1(14.9)$.

1-Phenyl-5-(p-bromophenyl)-2-thiohydantoin (22): Yield: $75.2 \%$. mp $205{ }^{\circ} \mathrm{C}$. TLC [methylene chloride: methanol (9:1)] Rf 0.26. ${ }^{\mathrm{H}} \mathrm{H}$ NMR (DMSO- $\left.d_{6}\right) \delta 7.56(\mathrm{~d} . J=$ $8.4 \mathrm{~Hz} .2 \mathrm{H}$. aromatic), 7.45 (d. $J=8.4 \mathrm{~Hz} .2 \mathrm{H}$. aromatic). $7.03(\mathrm{t}, J=8.4 \mathrm{~Hz}, 2 \mathrm{H}$. aromatic). $6.60(\mathrm{~d}, J=7.5 \mathrm{~Hz} .2 \mathrm{H}$. aromatic), 6.55 (t. $J=7.2 \mathrm{~Hz} .1 \mathrm{H}$, aromatic), $5.12(\mathrm{~s}, 1 \mathrm{H}$. $\mathrm{CH}) .3 .36(\mathrm{~s}, 1 \mathrm{H}, \mathrm{NH}) .{ }^{13} \mathrm{C}$ NMR (DMSO- $\left.d_{6}\right) \delta 172.83$ $(\mathrm{C}=\mathrm{O}, \mathrm{C}=\mathrm{S}), \quad 147.05 .138 .58 .131 .68 .130 .05 .129 .10$. $121.21,117.03,113.48$ (aromatic $\times 2$ ), $59.36(\mathrm{CH})$. FT-IR $(\mathrm{KBr}) \mathrm{cm}^{-1} 3407(\mathrm{NH}) .2992(\mathrm{CH}), 766(\mathrm{C}-\mathrm{Br}) . \mathrm{GC}-\mathrm{MS}$ : $\mathrm{m} / \mathrm{z} 348.22\left(\mathrm{M}^{-}\right), 169.0(100.0)$. $171.0(97.1)$. $261.0(72.4)$. $263.0(70.4) .90 .1(29.2)$.

1-(p-Methoxyphenyl)-5-phenyl-2-thiohydantoin (38): Yield: $92.7 \%$ mp $197.7-200.5{ }^{\circ} \mathrm{C}$. TLC [ $n$-hexane:ethyl acetate (2:1)] Rf 0.1 , [methylene chloride:methanol (9:1)] Rf 0.29 . ${ }^{1} \mathrm{H}$ NMR (DMSO- $d_{6}$ ) $\delta 7.50$ (d. $J=6.9 \mathrm{~Hz}, 2 \mathrm{H}$. aromatic), $7.37-7.28(\mathrm{~m}+\mathrm{d}, 3 \mathrm{H}$. aromatic), $6.64(\mathrm{~d}+\mathrm{d}, J=9.3$ and $9.3 \mathrm{~Hz} .4 \mathrm{H}$, aromatic). 5.01 (s. $\mathrm{HH}, \mathrm{CH}), 3.60$ (s, $3 \mathrm{H}$. $\mathrm{CH}_{3}$ ). 3.36 (s. IH. NH). ${ }^{13} \mathrm{C}$ NMR (DMSO- $\left.d_{6}\right) \delta 172.96$ $(\mathrm{C}=\mathrm{O} . \mathrm{C}=\mathrm{S}), 151.03,140.98,138.82 .128 .24 .127 .48 .127 .34$. $114.27,114.09$ (aronatic $\times 2), 60.46(\mathrm{CH}) .55 .10\left(\mathrm{CH}_{3}\right)$. FTIR $(\mathrm{KBr}) \mathrm{cm}^{-1} 3403(\mathrm{NH}), 1514(\mathrm{CO})$. GC-MS: $\mathrm{m} / \mathrm{z} 298.37$ $\left(\mathrm{M}^{+}\right) .196 .1(100.00) .211 .1$ (92.10). 167.1 (21.49). 210.2 (15.03). 197.1 (14.57).

1-( $p$-Methoxyphenyl)-5-(p-bromophenyl)-2-thiohydantoin (40): Yield: $93.3 \%, \mathrm{mp} 184.6-186.8^{\circ} \mathrm{C}$. TLC [ $n$-hexane :ethyl acetate (2:1)] Rf $0.1 .{ }^{1} \mathrm{H}$ NMR (DMSO- $\left.d_{6}\right) \delta 7.55$ (d. $J$ $=8.4 \mathrm{~Hz} .2 \mathrm{H}$. aromatic), 7.45 (d. $J=8.4 \mathrm{~Hz} .2 \mathrm{H}$, aromatic). 6.63 (dd. $J=9.3$ and $9 \mathrm{~Hz} .4 \mathrm{H}$. aromatic), 5.05 (s. $1 \mathrm{H} . \mathrm{CH}$ ). 3.60 (s. $3 \mathrm{H} . \mathrm{CH}_{3}$ ), 3.34 (s. $1 \mathrm{H}, \mathrm{NH}$ ). ${ }^{13} \mathrm{C}$ NMR (DMSO- $\left.d_{6}\right) \delta$ $172.54(\mathrm{C}=\mathrm{O} . \mathrm{C}=\mathrm{S}), 151.13 .140 .63 .138 .30,131.16,129.58$. 120.66, 114.24. 114.21 (aromatic $\times 2), 59.62(\mathrm{CH}), 55.05$ $\left(\mathrm{CH}_{3}\right)$. FT-IR $(\mathrm{KBr}) \mathrm{cm}^{-1} 3413(\mathrm{NH}) .1513$ (CO). 765 (C. Br). GC-MS: $\mathrm{m} / \mathrm{z} 377.26\left(\mathrm{M}^{+}\right) .291 .0(100.00), 289.0$ (97.51), 276.0 (87.35). 274.0 (86.73), $290.0(27.00)$.

5-Phenyl-1-(p-sulfamylphenyl)-2-thiohydantoin (45): Yield: $76.9 \%$. mp $242.0-248.0^{\circ} \mathrm{C}$, TLC [methylene chloride :methanol (9:1)] Rf 0.1. ${ }^{1} \mathrm{H}$ NMR (DMSO- $\left.d_{6}\right) \delta 7.48$ (d. $J=$ $9 \mathrm{~Hz}, 4 \mathrm{H}$, aromatic), 7.30-7.18 (m, 3H, aromatic), $6.77(\mathrm{~s}$. $\left.2 \mathrm{H} . \mathrm{NH}_{2}\right) .6 .57(\mathrm{~d} . J=9 \mathrm{~Hz}, 2 \mathrm{H}$, arontatic), $4.89(\mathrm{~s} .1 \mathrm{H}, \mathrm{CH})$. 3.25 (s. $1 \mathrm{H} . \mathrm{NH}$ ) ${ }^{13} \mathrm{C}$ NMR (DMSO- $\left.d_{6}\right) \delta 171.15(\mathrm{C}=\mathrm{O} . \mathrm{C}=\mathrm{S}$ ),
149.63, 140.09. 130.02, 127.88, 127.01, 126.95, 126.68, 111.61 (aromatic $\times 2), 60.56(\mathrm{CH})$. FT-IR $(\mathrm{NaCl}) \mathrm{cm}^{-1} 3367$ $(\mathrm{NH}), 3192\left(\mathrm{NH}_{2}\right) .1298\left(\mathrm{SO}_{2}\right)$. GC.MS: $\mathrm{m} / \mathrm{z} 347.42\left(\mathrm{M}^{-}\right)$, $270.2(100.00) .271 .2(52.55)$, 167.1 (37.52). $165.1(21.69)$. $77.1(16.51)$.

5-(p-Chlorophenyl)-1-( $p$-sulfamylphenyl)-2-thiohydantoin (46): Yield: $69.3 \%$. mp 200.9-204.2 ${ }^{\circ} \mathrm{C}$. TLC [methylene chloride:methanol (9:1)] Rf 0.1. ${ }^{1} \mathrm{H}$ NMR (DMSO- $d_{6}$ ) $\delta 7.44$ $(\mathrm{d}+\mathrm{d} . J=8.7$ and $8.4 \mathrm{~Hz} .4 \mathrm{H}$, aromatic), $7.23(\mathrm{~d} . J=8.4 \mathrm{~Hz}$. $2 \mathrm{H}$. aromatic), 6.62 (s. $2 \mathrm{H}$. NH.). 6.52 (d. $J=8.7 \mathrm{~Hz}, 2 \mathrm{H}$, aromatic). $4.94(\mathrm{~s}, \mathrm{lH}, \mathrm{CH}) .3 .23$ (s. $\mathrm{lH}, \mathrm{NH}) .{ }^{13} \mathrm{C}$ NMR $\left(\mathrm{DMSO}-d_{6}\right) \delta 171.84(\mathrm{C}=\mathrm{O} . \mathrm{C}=\mathrm{S})$. 149.34. 137.06. 132.35. $131.19,129.22 .128 .39,126.95 .111 .99$ (aromatic $\times 2) .58 .38$ (CH). FT-IR $(\mathrm{NaCl}) \mathrm{cm}^{-1} 3340(\mathrm{NH}), 3215\left(\mathrm{NH}_{2}\right) .1716$ $(\mathrm{C}=\mathrm{O}) .1301$ ( $\left.\mathrm{SO}_{2}\right) .721$ (C-Cl). GC-MS: $\mathrm{m} / \mathrm{z} 381.86\left(\mathrm{M}^{-}\right)$, $180.1(100.00)$. $181.1(86.85), 77.1(36.71), 51.1(13.17)$. $182.1(11.41)$.

Procedure for the 3-butyl-1,5-diphenylhydantoin (19). Methyl $\alpha$-anilinophenylacetate 7 (1.2 g. $4.98 \mathrm{mmol})$ and butyl isocyanate $(0.56 \mathrm{~mL}, 4.98 \mathrm{mmol})$ were dissolved in toluene $(10 \mathrm{~mL})$. The reaction mixture was then refluxed at $90{ }^{\circ} \mathrm{C}$ for $38 \mathrm{~h}$, cooled to room temperature and evaporated to remove the toluene. The residue obtained was purified by column chromatograply to give compound 19: TLC [ $n$ hexane:etlyyl acetate (4:1)] $\mathrm{Rf} 0.36$. ${ }^{1} \mathrm{H}$ NMR (DMSO- $d_{6}$ ) $\delta$ $7.48(\mathrm{~d}, j=7.8 \mathrm{~Hz} .2 \mathrm{H}$. aromatic). $7.35-7.24(\mathrm{~m}, 7 \mathrm{H}$, aromatic). 7.08 (t. $J=7.5 \mathrm{~Hz}, \mathrm{lH}$. aromatic). 5.43 (s. $1 \mathrm{H}$, $\mathrm{CH}), 3.65-3.59$ (m. $\left.2 \mathrm{H}, \mathrm{CH}_{2}\right) .1 .70-1.6 \mathrm{l}\left(\mathrm{m} .2 \mathrm{H}, \mathrm{CH}_{2}\right), 1.36$ (q. $J=7.5 \mathrm{~Hz} .2 \mathrm{H}, \mathrm{CH}_{3}$ ). 0.93 (t. $J=7.2 \mathrm{~Hz} .3 \mathrm{H}, \mathrm{CH}_{3}$ ). ${ }^{13} \mathrm{C}$ NMR (DMSO- $\left.d_{6}\right) \delta 170.03(\mathrm{C}=\mathrm{O} \times 2), 154.76,136.54$. $133.03,129.28,129.09,126.66 .124 .58,120.11 .113 .36$ (aromatic $\times 2$ ). $64.06(\mathrm{CH}) .39 .04\left(\mathrm{CH}_{2}\right) .30 .02\left(\mathrm{CH}_{2}\right), 19.92$ $\left(\mathrm{CH}_{2}\right) .13 .61\left(\mathrm{CH}_{3}\right)$. FT-IR $(\mathrm{NaCl}) \mathrm{cm}^{-1} 2957(\mathrm{CH}) .1774$ $(\mathrm{C}=\mathrm{O}), 1712(\mathrm{C}=\mathrm{O})$. GC-MS: m/z $308.38\left(\mathrm{M}^{-}\right)$. $180.1(100.0)$. $181.1(100.0), 182.1(100.0) .308 .2(100.0), 77.1(54.3)$.

General procedure for the compounds 24-27, 29-32, and 34-37. Methyl $\alpha$-( $p$-methoxyanilino)phenylacetate 10 $(0.5 \mathrm{~g} .1 .84 \mathrm{mmol})$ and molecular sieve $(4 \AA)$ were added to xylene $(10 \mathrm{~mL})$ and then triethylamine $(0.51 \mathrm{~mL}, 3.68$ nimol). and butyl isocyanate $(0.42 \mathrm{~mL}, 3.68 \mathrm{mmol})$ were added. The reaction mixture was refluxed $48 \mathrm{~h}$. cooled to room temperature and evaporated under reduced pressure. The residue obtained was crystallized and recrystallized from ethanol to yield a white solid.

3-Butyl-1-(p-methoxyphenyl)-5-phenylhydantoin (24): Yield: $0.61 \mathrm{~g}(62.3 \%)$. mp $101.8-103.7^{\circ} \mathrm{C}$. TLC [ $n$-hexane: ethyl acetate (2:1)] $\mathrm{Rf} 0.53$. ${ }^{1} \mathrm{H}$ NMR (DMSO- $\left.d_{6}\right) \delta 7.41$ $7.30(\mathrm{~m}+\mathrm{d} .7 \mathrm{H}$, aromatic). $6.86(\mathrm{~d}, J=9 \mathrm{~Hz}, 2 \mathrm{H}$. aromatic). 5.93 (s. $1 \mathrm{H} . \mathrm{CH}$ ). 3.68 (s. $3 \mathrm{H} . \mathrm{CH}_{3}$ ). $3.53-3.47\left(\mathrm{~m} .2 \mathrm{H}, \mathrm{CH}_{2}\right.$ ), $1.60-1.52\left(\mathrm{~m} .2 \mathrm{H} . \mathrm{CH}_{3}\right), 1.33-1.26\left(\mathrm{~m} .2 \mathrm{H}, \mathrm{CH}_{3}\right), 0.89(\mathrm{t}, J=$ $\left.7.5 \mathrm{~Hz} .3 \mathrm{H}, \mathrm{CH}_{3}\right) \cdot{ }^{13} \mathrm{C}$ NMR (DMSO- $\left.d_{6}\right) \delta 170.45(\mathrm{C}=\mathrm{O} \times 2)$, 156.21, 154.36. 133.84. 128.97, 128.85, 128.57. 127.27, 123.30. 113.92 (aromatic $\times 2$ ), $63.63(\mathrm{CH}), 55.06\left(\mathrm{CH}_{3}\right)$, $38.04\left(\mathrm{CH}_{2}\right), 29.44\left(\mathrm{CH}_{2}\right), 19.23\left(\mathrm{CH}_{2}\right), 13.39\left(\mathrm{CH}_{3}\right)$. FT-IR $(\mathrm{KBr}) \mathrm{cm}^{-1} 1770(\mathrm{C}=\mathrm{O}) .1710(\mathrm{C}=\mathrm{O}) .1514(\mathrm{CO})$. GC-MS: $\mathrm{m} / \mathrm{z} 338.41\left(\mathrm{M}^{-}\right), 338.3(100.00)$. 196.1 (78.65). 339.3 (53.26). $211.2(49.11) .212 .2(39.49)$. 
3-Pentyl-1-(p-methoxyphenyl)-5-phenylhydantoin (25): Yield: $41.3 \%$. mp $122.4-125.3{ }^{\circ} \mathrm{C}$. TLC [ $n$-hexane:ethyl acetate (2:1)] Rf 0.55. ${ }^{1} \mathrm{H}$ NMR (DMSO- $\left.d_{6}\right) \delta 7.41-7.30$ $(\mathrm{m}+\mathrm{d}, 7 \mathrm{H}$, aromatic $), 6.86(\mathrm{~d}, J=9.3 \mathrm{~Hz}, 2 \mathrm{H}$. aromatic). $5.93(\mathrm{~s} .1 \mathrm{H}, \mathrm{CH}), 3.68\left(\mathrm{~s}, 3 \mathrm{H} . \mathrm{CH}_{3}\right), 3.51-3.46\left(\mathrm{~m} .2 \mathrm{H}, \mathrm{CH}_{2}\right)$. $1.61-1.56\left(\mathrm{~m}, 2 \mathrm{H}, \mathrm{CH}_{-}\right), 1.31-1.24\left(\mathrm{~m},+\mathrm{H} . \mathrm{CH}_{-} \times 2\right) .0 .85(\mathrm{t}$. $\left.J=6.9 \mathrm{~Hz} .3 \mathrm{H}, \mathrm{CH}_{3}\right) \cdot{ }^{13} \mathrm{C}$ NMR (DMSO- $\left.d_{6}\right) \delta 170.43$ $(\mathrm{C}=\mathrm{O} \times 2), 156.19,154.34,133.84 .128 .98 .128 .83 .128 .57$. $127.27,123.27,113.92$ (aromatic $\times 2), 63.61(\mathrm{CH}), 55.06$ $\left(\mathrm{CH}_{3}\right), 38.29\left(\mathrm{CH}_{2}\right), 28.12\left(\mathrm{CH}_{2}\right), 26.96\left(\mathrm{CH}_{3}\right), 21.53\left(\mathrm{CH}_{2}\right)$. $13.72\left(\mathrm{CH}_{3}\right)$. FT-IR $(\mathrm{KBr}) \mathrm{cm}^{-1} 1771(\mathrm{C}=\mathrm{O}) .1711(\mathrm{C}=\mathrm{O})$. 1514 (CO). GC.MS: $\mathrm{m} / \mathrm{z} 352.43\left(\mathrm{M}^{-}\right), 352.3(100.00), 196.1$ $(70.30), 353.3(55.57) .212 .2(44.00), 211.2(42.03)$.

3-Phenyl-1-( $p$-methoxyphenyl)-5-phenylhydantoin (26): Yield: $32.3 \% \mathrm{mp} 145.8-148.0^{\circ} \mathrm{C}$. TLC [ $n$-hexane:ethyl acetate (2:1)] $\mathrm{Rf} 0.4 .{ }^{1} \mathrm{H}$ NMR (DMSO- $d_{6}$ ) $\delta$ 7.56-7.35 $(\mathrm{m}+\mathrm{d}, 12 \mathrm{H}$, aromatic $), 6.90(\mathrm{~d} . J=9 \mathrm{~Hz}, 2 \mathrm{H}$. aromatic $), 6.07$ (s. $\mathrm{IH} . \mathrm{CH}) .3 .69$ (s. $3 \mathrm{H}, \mathrm{CH}_{3}$ ). ${ }^{13} \mathrm{C}$ NMR (DMSO- $\left.d_{6}\right) \delta$ $169.61(\mathrm{C}=\mathrm{O} \times 2), 156.44,153.39,133.76,131.78,128.86$. 128.71. 128.38, 127.68, 127.05. 126.93, 123.80, 113.96 (aromatic $\times 3), 63.94(\mathrm{CH}), 55.07\left(\mathrm{CH}_{3}\right)$. FT-IR $(\mathrm{KBr}) \mathrm{cm}^{-1}$ $1778(\mathrm{C}=\mathrm{O}), 1716(\mathrm{C}=\mathrm{O}) .1512(\mathrm{CO})$. GC-MS: $\mathrm{m} / \mathrm{z} 358.40$ $\left(\mathrm{M}^{+}\right) .358 .2(100.00) .211 .2(87.82) .196 .1(86.25) .359 .2$ (53.89), 167.1 (19.48).

3-Benzyl-1-( $p$-methoxyphenyl)-5-phenylhydantoin (27): Yield: $55.2 \%, \mathrm{mp} 118.9-121.6{ }^{\circ} \mathrm{C}$. TLC [n-hexane:ethyl acetate $(2: 1)]$ Rf 0.45 . ${ }^{1} \mathrm{H}$ NMR (DMSO- $d_{6}$ ) $\delta$ 7.43-7.29 $(\mathrm{m}+\mathrm{d}, 12 \mathrm{H}$, aromatic), 6.87 (d. $J=9 \mathrm{~Hz}, 2 \mathrm{H}$. aromatic), 6.05 (s. $\mathrm{lH}$. CH) 4.70 (s. $\left.2 \mathrm{H} . \mathrm{CH}_{2}\right) .3 .68\left(\mathrm{~s}, 3 \mathrm{H}, \mathrm{CH}_{3}\right) .{ }^{13} \mathrm{C}$ NMR $\left(\mathrm{DMSO}-d_{6}\right) \delta 170.31(\mathrm{C}=\mathrm{O} \times 2), 156.30 .154 .17,136.25$. 133.63. 128.86, 128.64. 128.50. 127.46, 127.30. 123.40. 113.94 (aronatic $\times 3), 63.82(\mathrm{CH}), 55.07\left(\mathrm{CH}_{3}\right), 41.79\left(\mathrm{CH}_{3}\right)$. FT-IR $(\mathrm{KBr}) \mathrm{cm}^{-1} 1777(\mathrm{C}=\mathrm{O}), 1713(\mathrm{C}=\mathrm{O}) .1515(\mathrm{CO})$. GC-MS: $\mathrm{m} / \mathrm{z} 372.42\left(\mathrm{M}^{+}\right) .372 .1$ (100.00). 196.1 (38.26). $211.1(28.23), 373.1(26.95) .91 .1(25.55)$.

3-Butyl-1-( $p$-methoxyphenyl)-5-( $p$-chlorophenyl)hydantoin (29): Yield: $67.2 \%, \mathrm{mp} 93.6-95.2^{\circ} \mathrm{C}$. TLC [ $n$-hexane: ethyl acetate (2:1)] Rf 0.30 . ${ }^{1} \mathrm{H}$ NMR (DMSO- $\left.d_{6}\right) \delta 7.44-$ 7.38 (m. $6 \mathrm{H}$ aromatic). 6.88 (d. $J=6.9 \mathrm{~Hz} 2 \mathrm{H}$ aromatic). 5.98 (s. $1 \mathrm{H} . \mathrm{CH}) .3 .69$ (s. $\left.3 \mathrm{H}, \mathrm{CH}_{3}\right), 3.52-3.45$ (m. $2 \mathrm{H}, \mathrm{CH}_{2}$ ). $1.59-1.54\left(\mathrm{~m}, 2 \mathrm{H}, \mathrm{CH}_{2}\right) .1 .33-1.28\left(\mathrm{~m}, 2 \mathrm{H}, \mathrm{CH}_{2}\right), 0.89(\mathrm{t} . J=$ $\left.7.5 \mathrm{~Hz}, 3 \mathrm{H} . \mathrm{CH}_{3}\right) .{ }^{13} \mathrm{C}$ NMR (DMSO- $\left.d_{6}\right) \delta 170.56(\mathrm{C}=\mathrm{O} \times 2)$. 156.72. 154.72, 133.74. 133.28. 129.68, 129.33. 129.25 . 123.73. 114.42 (aromatic $\times 2$ ). $63.35(\mathrm{CH}), 55.52\left(\mathrm{CH}_{3}\right)$. $38.57\left(\mathrm{CH}_{2}\right) .29 .86\left(\mathrm{CH}_{2}\right) .19 .68\left(\mathrm{CH}_{2}\right) .13 .84\left(\mathrm{CH}_{3}\right)$. FT-IR $\left(\mathrm{NaCl} \mathrm{cm}^{-1} 1771(\mathrm{C}=\mathrm{O}) .1712(\mathrm{C}=\mathrm{O}) .1514(\mathrm{CO}), 831(\mathrm{Cl})\right.$. GC-MS: $\mathrm{m} / \mathrm{z} 372.85\left(\mathrm{M}^{-}\right) 230.1(100.0), 245.1(64.81)$. $246.1(50.87), 149.1(35.40)$. $232.1(34.45)$.

3-Pentyl-1-( $p$-methoxyphenyl)-5-( $p$-chlorophenyl)hydantoin (30): Yield: $79.4 \%, \mathrm{mp} 97.4-98.6^{\circ} \mathrm{C}$. TLC [ $n$-hexane :ethyl acetate (2:1)] Rf 0.35. ${ }^{1} \mathrm{H}$ NMR (DMSO- $d_{6}$ ) $\delta 7.44$ 7.35 ( $\mathrm{m}, 6 \mathrm{H}$, aromatic). 6.88 (d, $J=6.9 \mathrm{~Hz} 2 \mathrm{H}$, aromatic). 5.99 (s. $1 \mathrm{H} . \mathrm{CH}) .3 .69$ (s. $\left.3 \mathrm{H} . \mathrm{CH}_{3}\right), 3.51-3.48$ (m. $2 \mathrm{H}_{,} \mathrm{CH}_{2}$ ). $1.61-1.56\left(\mathrm{~m}, 2 \mathrm{H} . \mathrm{CH}_{2}\right), 1.31-1.24\left(\mathrm{~m}, 4 \mathrm{H}, \mathrm{CH}_{2}+\mathrm{CH}_{2}\right), 0.85$ $\left(\mathrm{t}, J=6.9 \mathrm{~Hz}, 3 \mathrm{H} . \mathrm{CH}_{3}\right.$ ), ${ }^{13} \mathrm{C}$ NMR (DMSO- $\left.d_{6}\right) \delta 170.09$ $(\mathrm{C}=\mathrm{O} \times 2), 156.26,154.25,133.29 .132 .84 .129 .23 .128 .86$. 128.81, 123.24, 113.97 (aromatic $\times 2$ ) $, 62.88(\mathrm{CH}), 55.07$
$\left(\mathrm{CH}_{3}\right) .38 .55\left(\mathrm{CH}_{2}\right) .28 .13\left(\mathrm{CH}_{2}\right), 26.95\left(\mathrm{CH}_{2}\right), 21.53\left(\mathrm{CH}_{2}\right)$, $13.72\left(\mathrm{CH}_{3}\right)$. FT-IR $(\mathrm{NaCl}) \mathrm{cm}^{-1} 1772(\mathrm{C}=\mathrm{O}) .1712(\mathrm{C}=\mathrm{O})$, 1514 (CO). 828 (Cl). GC-MS: $\mathrm{m} / \mathrm{z} 386.88\left(\mathrm{M}^{+}\right) 230.1(100.0)$, 246.1 (65.19). 245.1 (62.04). 149.1 (40.78), 232.1 (33.94).

3-Phenyl-1-( $p$-methoxyphenyl)-5-( $p$-chlorophenyl)hydantoin (31): Yield: $62.1 \% \mathrm{mp} 180.2-183.2^{\circ} \mathrm{C}$, TLC [ 7 -hexane: ethyl acetate (2:1)] Rf 0.48. ${ }^{~} \mathrm{H}$ NMR (DMSO- $\left.d_{6}\right) \delta 7.56-$ $7.42(\mathrm{~d}+\mathrm{m} .11 \mathrm{H}$, aromatic). $6.91(\mathrm{~d} . J=9 \mathrm{~Hz} .2 \mathrm{H}$. aromatic), 6.11 (s. $1 \mathrm{H} . \mathrm{CH}) .3 .70\left(\mathrm{~s}, 3 \mathrm{H}, \mathrm{CH}_{3}\right.$ ). ${ }^{13} \mathrm{C}$ NMR (DMSO- $\left.d_{\mathrm{k}}\right) \delta$ $169.74(\mathrm{C}=\mathrm{O} \times 2) .156 .98 .153 .78 .133 .91,132.20,130.12$. 129.32. 127.49. 124.26, 114.48 (aromatic $\times 3$ ). $63.70(\mathrm{CH})$, $55.55\left(\mathrm{CH}_{3}\right)$. FT-IR $(\mathrm{KBr}) \mathrm{cm}^{-1} 1777(\mathrm{C}=\mathrm{O}), 172 \mathrm{l}(\mathrm{C}=\mathrm{O})$. $1513(\mathrm{CO}) .831(\mathrm{Cl})$.

3-Benzyl-1-( $p$-methoxyphenyl)-5-(p-chlorophenyl)hydantoin (32): Yield: $89.6 \%, \mathrm{mp} 147.6-148.8{ }^{\circ} \mathrm{C}$. TLC [ $n$ hexane:ethyl acetate (2:1)] Rf 0.37. ${ }^{1} \mathrm{H}$ NMR (DMSO- $c_{6}$ ) $\delta$ $7.69-7.24(\mathrm{~m} .9 \mathrm{H}$, aromatic), $7.26(\mathrm{~d} . J=6.9 \mathrm{~Hz} 2 \mathrm{H}$. aromatic), 6.85 (d. $J=6.9 \mathrm{~Hz} 2 \mathrm{H}$, aromatic). 6.05 (s. $1 \mathrm{H}, \mathrm{CH}$ ), 4.74 (s, $\left.2 \mathrm{H}_{2}, \mathrm{CH}_{2}\right) .3 .69$ (s. $3 \mathrm{H}_{2} \mathrm{CH}_{3}$ ). ${ }^{13} \mathrm{C}$ NMR (DMSO- $d_{6}$ ) $\delta 171.62(\mathrm{C}=\mathrm{O} \times 2), 158.01,154.32 .136 .50 .135 .63 .134 .02$, $128.94,128.91 .128 .52$. 127.92, 127.80, 127.18, 114.27 (aromatic $\times 3) .88 .10(\mathrm{CH}) .55 .50\left(\mathrm{CH}_{3}\right), 42.22\left(\mathrm{CH}_{2}\right)$. FT-IR $(\mathrm{NaCl}) \mathrm{cm}^{-1} 1773(\mathrm{C}=\mathrm{O}) .1713(\mathrm{C}=\mathrm{O}), 1513(\mathrm{CO}), 828(\mathrm{Cl})$. GC-MS: $\mathrm{m} / \mathrm{z} 406.87\left(\mathrm{M}^{+}\right) 230.1(100.0), 91.1(93.79), 245.1$ $(83.25)$. 166.0 (61.75), 246.1 (38.77).

3-Butyl-1-( $p$-methoxyphenyl)-5-( -bromophenyl)hydantoin (34): Yield: $56.7 \%, \mathrm{mp} 102.7 \cdot 104.5^{\circ} \mathrm{C}$. TLC [ $n$ hexane:ethyl acetate (2:1)] Rf 0.30. ${ }^{1} \mathrm{H}$ NMR (DMSO- $\left.d_{6}\right) \delta$ 7.55 (d. $J=6.6 \mathrm{~Hz} 2 \mathrm{H}$, aromatic), 7.39 (d. $J=6.6 \mathrm{~Hz} 2 \mathrm{H}$, aromatic). $7.30(\mathrm{~d} . J=6.6 \mathrm{~Hz} 2 \mathrm{H}$, aromatic). 6.88 (d. $J=6.9$ $\mathrm{Hz} 2 \mathrm{H}$, aromatic). 5.97 (s. $1 \mathrm{H}, \mathrm{CH}) .3 .69\left(\mathrm{~s}, 3 \mathrm{H}, \mathrm{CH}_{3}\right) .3 .52-$ 3.49 (m. $\left.2 \mathrm{H} . \mathrm{CH}_{2}\right) .1 .59-1.54$ (m. $\left.2 \mathrm{H} . \mathrm{CH}_{2}\right) .1 .30-1.27$ (m, $\left.2 \mathrm{H} . \mathrm{CH}_{3}\right) .0 .90\left(\mathrm{t} . J=7.2 \mathrm{~Hz}, 3 \mathrm{H}, \mathrm{CH}_{3}\right) \cdot{ }^{13} \mathrm{C}$ NMR (DMSOd $d_{6} \delta 170.49(\mathrm{C}=\mathrm{O} \times 2), 156.71,154.72 .133 .70,132.25$, $129.98,129.24$. 123.70. 122.35, 114.42 (aromatic $\times 2$ ), 63.41 $(\mathrm{CH}), 55.52\left(\mathrm{CH}_{3}\right) .38 .57\left(\mathrm{CH}_{2}\right), 29.86\left(\mathrm{CH}_{2}\right) .19 .68\left(\mathrm{CH}_{2}\right)$, $13.84\left(\mathrm{CH}_{3}\right)$. FT-IR $(\mathrm{NaCl}) \mathrm{cm}^{-1} 1771(\mathrm{C}=\mathrm{O}), 1711(\mathrm{C}=\mathrm{O})$, 1514 (CO). 830 (Br). GC-MS: $\mathrm{m} / \mathrm{z} 417.30\left(\mathrm{M}^{-}\right) 276.0$ (100.0), $274.0(99.63) .291 .0(65.28) .289 .0(61.55) .290 .0(52.65)$.

3-Pentyl-1-( $p$-methoxyphenyl)-5-( $p$-bromophenyl)hydantoin (35): Yield: $54.8 \%$ mp $91.7-93.9^{\circ} \mathrm{C}$. TLC [ 7 -hexane ethyl acetate (2:1)] Rf $0.40 .{ }^{1} \mathrm{H}$ NMR (DMSO- $\left.d_{6}\right) \delta 7.55$ (d, $J=8.7 \mathrm{~Hz} 2 \mathrm{H}$, aromatic), $7.38(\mathrm{t}, J=8.7 \mathrm{~Hz} 2 \mathrm{H}$. aromatic), 7.27 (q. $J=8.4 \mathrm{~Hz} 2 \mathrm{H}$. aromatic). 6.86 (t. $J=9 \mathrm{~Hz} 2 \mathrm{H}$. aromatic). 5.97 (s. $1 \mathrm{H} . \mathrm{CH}$ ). 3.69 (s. $\left.3 \mathrm{H} . \mathrm{CH}_{3}\right) .3 .51-3.47$ (m, $\left.2 \mathrm{H} . \mathrm{CH}_{3}\right), 1.61-1.58\left(\mathrm{~m}, 2 \mathrm{H} . \mathrm{CH}_{3}\right), 1.31-1.26\left(\mathrm{~m}, 4 \mathrm{H} . \mathrm{CH}_{2}+\right.$ $\left.\mathrm{CH}_{2}\right) .0 .86\left(\mathrm{t}, J=6.6 \mathrm{~Hz}, 3 \mathrm{H} . \mathrm{CH}_{3}\right) \cdot{ }^{13} \mathrm{C}$ NMR (DMSO- $\left.d_{6}\right) \delta$ $171.65(\mathrm{C}=\mathrm{O}) . \quad 170.09(\mathrm{C}=\mathrm{O}) . \quad 156.70, \quad 154.46, \quad 133.70$, $132.24,129.98 .128 .81,127.64 .123 .68,114.42$ (aromatic 2). $63.40(\mathrm{CH}) .55 .50\left(\mathrm{CH}_{3}\right) .28 .58\left(\mathrm{CH}_{2}\right), 27.58\left(\mathrm{CH}_{2}\right)$. $27.40\left(\mathrm{CH}_{2}\right) .21 .98\left(\mathrm{CH}_{2}\right) .14 .17\left(\mathrm{CH}_{3}\right)$. FT-IR $(\mathrm{NaCl}) \mathrm{cm}^{-1}$ $1772(\mathrm{C}=\mathrm{O}) .1712(\mathrm{C}=\mathrm{O}) .1514(\mathrm{CO}) .827(\mathrm{Br}) . \mathrm{GC}-\mathrm{MS}$ : $\mathrm{m} / \mathrm{z} 431.33\left(\mathrm{M}^{+}\right) 274.0(100.0), 276.0(98.20), 290.0(69.05)$. $291.0(67.19), 289.0(60.88)$.

3-Phenyl-1-( $p$-methoxyphenyl)-5-(pbromophenyl)hydantoin (36): Yield: $30.4 \% \mathrm{mp} 189.5-190.1{ }^{\circ} \mathrm{C}$. TLC [ 7 -hexane: ethyl acetate (2:1)] Rf 0.48 . 'H NMR (DMSO- $d_{6}$ ) $\delta 7.60-$ 
$7.29(\mathrm{~m}+$ d. $11 \mathrm{H}$. aromatic). 6.91 (d. $J=8.9 \mathrm{~Hz} .2 \mathrm{H}$. aromatic), 6.10 (s. $1 \mathrm{H}, \mathrm{CH}$ ), $3.70\left(\mathrm{~s}, 3 \mathrm{H} . \mathrm{CH}_{3}\right),{ }^{13} \mathrm{C} \mathrm{NMR}$ $\left(\mathrm{DMSO}-d_{6}\right) \delta 169.69(\mathrm{C}=\mathrm{O} \times 2), 156.97 .153 .79,133.65$. 132.25. 130.44, 129.34. 127.52, 124.22. 114.48 (aromatic $\times 3) .63 .74(\mathrm{CH}), 55.55\left(\mathrm{CH}_{3}\right)$. FT-IR $(\mathrm{KBr}) \mathrm{cm}^{-1} 1777$ $(\mathrm{C}=\mathrm{O}), 1721(\mathrm{C}=\mathrm{O}), 151+(\mathrm{CO}) .831(\mathrm{Br}) . \mathrm{GC}-\mathrm{MS}: \mathrm{m} / \mathrm{z}$ $437.29\left(\mathrm{M}^{+}\right) 256.1(100.0)$, $120.1(56.40)$. $228.1(40.36)$. 258.1 (34.73), 92.1 (32.19).

3-Benzyl-1-( $p$-methoxyphenyl)-5-( $p$-bromophenyl)hydantoin (37): Yield: $87.7 \%$. mp $139.5-1+1.0^{\circ} \mathrm{C}$. TLC [nhexane:ethyl acetate (2:1)] $\operatorname{Rf} 0.40 .{ }^{1} \mathrm{H}$ NMR (DMSO- $\left.d 6\right)$ 8.24 (s, $\mathrm{lH} . \mathrm{OH}$ ), 7.55 (d. $J=6.9 \mathrm{~Hz} 2 \mathrm{H}$. aromatic), 7.39 7.35 (m, $7 \mathrm{H}$. aromatic), 7.26 (d, $J=6.6 \mathrm{~Hz} 2 \mathrm{H}$, aromatic). $6.85\left(\mathrm{~d}, J=6.9 \mathrm{~Hz} 2 \mathrm{H}\right.$. aromatic), 4.73 (s. $\left.2 \mathrm{H}, \mathrm{CH}_{2}\right), 3.69$ (s. $\left.3 \mathrm{H}, \mathrm{CH}_{3}\right){ }^{13} \mathrm{C}$ NMR (DMSO- $\left.d_{6}\right) \delta 171.57(\mathrm{C}=\mathrm{O} \times 2) .158 .01$. 154.32. 136.49, 136.05. 131.84. 128.95, 128.82. 127.95. 127.91. 127.76. 127.18. 122.731. 114.27 (aromatic $\times 3$ ). $88.17(\mathrm{CH}), 55.50\left(\mathrm{CH}_{3}\right), 42.22\left(\mathrm{CH}_{3}\right)$. FT-IR $(\mathrm{NaCl}) \mathrm{cm}^{-1}$ $1773(\mathrm{C}=\mathrm{O}), 1713(\mathrm{C}=\mathrm{O}) .1513(\mathrm{CO}) .828(\mathrm{Br}) . \mathrm{GC}-\mathrm{MS}$ : $\mathrm{m} / \mathrm{z} 451.32\left(\mathrm{M}^{-}\right) 248.1(100.0)$, 171.1 (36.00). 77.1 (29.97). 105.1 (29.62), $115.1(28.59)$.

General pocedure for the compounds 39, +1 . Methyl $\alpha$ ( $p$-methoxyanilino)pheny lacetate $10(1.0 \mathrm{~g}, 3.69 \mathrm{mmol})$ and molecular sieve $(4 \AA)$ were added to xylene $(10 \mathrm{~mL})$ and then triethylanine $(1.03 \mathrm{~mL}, 7.38 \mathrm{mmol})$, benzyl isothiocyanate $(0.98 \mathrm{~mL}, 7.38 \mathrm{mmol})$ were added. The reaction mixture was refluxed for $48 \mathrm{~h}$, cooled to room temperature and then evaporated under reduced pressure. The residue obtained was crystallized and recrystallized from ethanol to yield white solid

3-Benzyl-1-( $p$-methoxyphenyl)-5-phenyl-2-thiohydantoin (39): Yield: $0.7 \mathrm{~g}(48.6 \%)$. mp 119.1-121.1 ${ }^{\circ} \mathrm{C}$, TLC $[n$ hexane:ethyl acetate (2:1)] Rf $0.4 .{ }^{1} \mathrm{H}$ NMR (DMSO- $d_{6}$ ) $\delta$ $7.42-7.22(\mathrm{~m}+\mathrm{d}, 12 \mathrm{H}$, aromatic), $6.89(\mathrm{~d}, J=9 \mathrm{~Hz} .2 \mathrm{H}$. aromatic), $6.12(\mathrm{~s}, \mathrm{lH}, \mathrm{CH}) .5 .08\left(\mathrm{~d} . J=3.3 \mathrm{~Hz} .2 \mathrm{H} . \mathrm{CH}_{2}\right)$. 3.71 (s. $\left.3 \mathrm{H} . \mathrm{CH}_{3}\right) .{ }^{13} \mathrm{C}$ NMR (DMSO- $\left.d_{6}\right) \delta 171.70(\mathrm{C}=\mathrm{O} . \mathrm{C}=\mathrm{S})$, 158.10. 136.04, 132.82. 129.69. 129.00, 128.47. 128.38. 128.19.127.78. 127.63. 127.37.113.86 (aromatic $\times 3$ ). 68.00 (CH). $55.13\left(\mathrm{CH}_{3}\right), 44.70\left(\mathrm{CH}_{2}\right)$. FT-IR $(\mathrm{KBr}) \mathrm{cm}^{-1} 1752$ $(\mathrm{C}=\mathrm{O}) .1513$ (CO). GC-MS: $\mathrm{m} / \mathrm{z} 388.49\left(\mathrm{M}^{-}\right) .388 .2(100.00)$, 212.2 (41.03). 387.2 (29.02). 389.2 (27.82). 91.1 (26.68).

3-Benzyl-1-( $p$-methoxyphenyl)-5-( $p$-bromophenyl-2thiohydantoin (41): Yield: $27.2 \%$ mp $188.7-191.0^{\circ} \mathrm{C}$. TLC [ $n$-hexane ethyl acetate $(2: 1)]$ Rf $0.35 .{ }^{1} \mathrm{H}$ NMR (DMSO- $\left.d_{6}\right) \delta 7.56-7.42(\mathrm{~d}+\mathrm{m} .1 \mathrm{lH}$. aromatic), $6.9 \mathrm{l}$ (d. $J=9$ $\mathrm{Hz}, 2 \mathrm{H}$, aromatic). 6.11 (s, $1 \mathrm{H} . \mathrm{CH}), 5.08\left(\mathrm{~s}, 2 \mathrm{H} . \mathrm{CH}_{2}\right.$ ). $3.70\left(\mathrm{~s}, 3 \mathrm{H}, \mathrm{CH}_{3}\right) .{ }^{13} \mathrm{C}$ NMR (DMSO- $\left.d_{6}\right) \delta 169.74(\mathrm{C}=\mathrm{O}$. $\mathrm{C}=\mathrm{S}) .156 .98,153.78 .133 .91,132.20 .130 .12,129.32$. 127.49, 124.26, 114.48 (aromatic $\times 3), 63.70(\mathrm{CH}), \mathbf{5 5 . 5 5}$ $\left(\mathrm{CH}_{3}\right), 44.50\left(\mathrm{CH}_{2}\right)$. FT-IR $(\mathrm{KBr}) \mathrm{cm}^{-1} 1777(\mathrm{C}=\mathrm{O}) .1720$ $(\mathrm{C}=\mathrm{O}), 1513(\mathrm{CO}), 83 \mathrm{l}(\mathrm{C}-\mathrm{Br})$
Acknowledgment. This work was supported by the Basic Research Program of the Korean Science \& Engineering Foundation (Grant No. R04-2002-000-00079-0).

\section{References}

1. (a) Hardman. . G. G.: Limbird. L. E. In Goodntam and Gilmon's The Phamacological Basis of Therapettics. 10th ed.: MeGraw-Hill: New York. 2001: pp 688-731. (b) Lombardino, G. Nonsteroidal Antiinflammony Drugs, John Wiely \& Sons: New York, 1985.

2. Allison. M. C.: Howatson. A. G.: Torrance. C. T.: Lee. F. D.: Russell. R. I. G. N. Engl, J. Med. 1992. 327. 749.

3. (a) Fu. . . Y.: Masfferrer. T. L.: Seibert. K.: Raz. A.: Needleman1. P. J. Biol Chem. 1990, 265, 16737. (b) Vane. J.; Botting, J.: Botting. R. Inproved Kon-stemid Antinflammatory Dings CON-2 Enzyme Inhibitors, Kluwer Academic Publishers and William Harvey Press: London. 1996.

4. Ha. T.: Neilson. K. Proc. Nath Acad Sci. U.S.4 1992.89.7384.

5. Xie. W.: Chipman. J. G: Rohertsont. D. L.: Erikson1. R. L.: Simmons. D. L. Proc. Natl Acad Sci. U.S.A. 1991. 88, 2692.

6. Kujubu, D. A:; Fletcher, B. S.: Varnum, B. C.: Lim, R. W: Herschman, H. R. J. Biol. Chem. 1991. 266. 12866.

7. Masferrer. J. L.: Seibert. K.: Zweifel. B.: Needleman. P. Proc. Natl. Acad Sci. U.S.A. 1992. 89. 3917.

8. Futaki. N.: Yoshikawa. K.: Hamasaka. Y.: Arai. I.: Higuchi. S.: Iizuka, H.: Otomo. S. Gen. Phamacol 1993. 24, 105.

9. Futaki, N.: Takahashi. S.: Yokoyama. M.: Arai. I.: Higuchi, S.; Otomo. S. Prostaglandins 1994, $47,55$.

10. Gans. K. R: Galnraith, W: Romans. R. J.: Haber, S. B.: Kerr. J S.: Schnidt. W. K.: Smith. C.: Hewes. W. E.: Ackermant. N. R. J. Phamacol. Exp. Ther. 1990. 254. 180.

11. Penning. T. D.: Tally. J. T.: Bertenshaw. S. R.: Carter. T. S.: Collins, P. W.: Doctor. S.: Graneto, M. J.: Lee. L. F; Veenhuizen. A. W. Zhang, Y. Y.: Isakson, P. C. J. Hed. Chem 1997, 10. 1347.

12. Prasit. P.: Wang. Z.: Brideau. C.: Chan. C.-C.: Charleson. S.: Cromlish. W.: Ethier. D.: Evans. I. F.: Ford-Hutchinson. A. W: Gauthier. T. Y: Gordon. R.: Forrest. M.: Visco. D.: Patrick. D. Bioorg. 1 fed. Chem. Lett. 1999. 9, 1773.

13. Talley, J. J.; Brown. D. L.: Carter. J. S ; Graneto. M. J.; Koboldt. C. M.: Masferrer. J. L. Perkins. W. E.: Rogers, R. S.: Shaffer. A. F.: Zhang. Y. Y.: Zweifel. B. S.: Seibert. K. J. Hed Chent 2000. $\$ 3.775$.

14. Kwont. S. K.: Jung. H. W: Park. M. S. Korem J. Hed. Chen. $1995.5,166$.

15. Wolfe, M. M.; Lichtenstein, D. R.; Singh. G. N. Engl. J. Mfed. 1999. 340,1888

16. Mukherjee. D: Nissen1. S. E.: Topol. E. T. JAML4 2001. 286.954.

17. Gaetano. G.: Donati. M. B.: Cerletti. C. Thends Phom. Sci. 2003. $2+.245$.

18. Hwang. K. J.: Lee, S. J.; Kim, B. T: Raucher, S. Bull. Koman Chem. Soc. 2006, 27(6).933.

19. Park. M. S.: Park. H. S. Yakhak Hoeji 2003. 47. 276

20. Kirk-Othmer Encwiopedia of Chentical Technologis. 2nd ed: Interscience: New York-London-Sydney. 1966: Vol 11. pp 141161 .

21. (a) Ware. E. Chem Rev 1950. 411. (b) Li, J. P. Org. Chem 1975 10. 3414. (c) Kirk-Othmer Encyclopedia of Chemical Techolog: 3nd ed.: Interscience: New York-London Sydney. 1979; Vol 12 , pp 692-708. 